\title{
DIRECT COST OF DRUG AND PRESCRIBING PATTERN IN THE OUTPATIENT SERVICES OF A TEACHING HOSPITAL IN MALAYSIA
}

\begin{abstract}
SIT WAI LEE ${ }^{1,2 *}$, SYED MOHAMED ALJUNID ${ }^{1,3}$
${ }^{1}$ International Centre for Case Mix and Clinical Coding, Faculty of Medicine, Universiti Kebangsaan Malaysia, Kuala Lumpur, Malaysia. ${ }^{2}$ Malaysian Health Technology Assessment Section, Medical Development Division, Ministry of Health Malaysia, Putrajaya, Malaysia.

${ }^{3}$ Department of Health Policy and Management, Faculty of Public Health, Kuwait University, Kuwait. Email: leesitwai@gmail.com
\end{abstract}

Received: 02 September 2017, Revised and Accepted: 29 March 2018

ABSTRACT

Objective: This study aims to impute the direct drug cost and to assess the prescribing pattern on patients getting dyslipidemia drugs at outpatients' Pharmacy Department in Universiti Kebangsaan Malaysia Medical Centre (UKMMC).

Methods: Patients receiving at least one dyslipidemia drug were randomly selected from UKMMC pharmacy electronic prescribing system from January 2012 to December 2012. The patients' gender, age, and ethnic, and all the drugs received by the patients during the visits were recorded. The brand name of the drugs and the cost was recorded for the calculation of generic drug prescription rate (GDPR) and direct drug cost.

Results: A total of 380 patients were randomly selected from the system. There were 829 prescriptions with the total amount of 5512 drugs prescribed. From the total of drugs, the number of generic drugs prescribed was 3967 (72\%). The percentage of patients with GDPR of more than $75 \%$ for this study was $48 \%(\mathrm{n}=183)$. The total drug cost for all 380 patients was RM240,244. The mean cost for each patient was RM637 (Range: RM1.20RM27,972.30) and the median cost was RM233. The total formulary dyslipidemia drugs cost was RM11,003. The mean formulary dyslipidemia drugs cost for each patient was RM38 (Range: RM1.20-RM810), and the median cost was RM13.50. Predictors of high drug cost were GDPR of $\leq 75 \%$ and a number of drugs per prescription of $>10$.

Conclusion: This study could contribute toward short- and long-term strategies to reduce the drug cost expenditure of dyslipidemia drugs in the hospital.

Keywords: Drug cost, Generic drugs prescribing rate, Dyslipidemia drugs.

(c) 2018 The Authors. Published by Innovare Academic Sciences Pvt Ltd. This is an open access article under the CC BY license (http://creativecommons. org/licenses/by/4. 0/) DOI: http://dx.doi.org/10.22159/ajpcr.2018.v11i7.22331

\section{INTRODUCTION}

Non-communicable diseases (NCD) or chronic diseases are the type of diseases that progress slowly and normally required extensive and proper management. The World Health Organization classified four types of NCD that cause most death as cardiovascular diseases (CVD), followed by cancers, respiratory diseases, and diabetes. These four groups of diseases account for around $80 \%$ of all NCDs deaths. Dyslipidemia is defined as the abnormal value of lipid profile. It has been identified as one of the main risk factors for CVD [1]. The overall prevalence of dyslipidemia cases (known and undiagnosed) among adults of 18 years and above was $47.7 \%$ (95\% confidence intervals [CI]: 46.5, 48.9). There was a general increasing trend in the prevalence of dyslipidemia with age, from $22.0 \%(95 \% \mathrm{CI}: 18.8,25.7)$ in the $18-19$ years age group, reaching a peak of $68.8 \%(95 \% \mathrm{CI}: 65.9,71.5)$ among the 55-59 years age group [2].

The high prevalent cases of dyslipidemia have alarmed the public funded government to have better fund management to prevent the development of chronic cases. It is the aim of the government to provide value-based medicine to the public. Malaysia has introduced Malaysian National Medicines Policy since the endorsement in the year 2006 by the Malaysian Cabinet [3]. One of the government policies in ensuring affordability of the drugs is by promoting usage of generic drugs in Malaysia. There has been a great debate for the appropriateness of generic substitution of originator drugs to reduce the cost of the drugs. A study was done to compare the prescribing pattern and drug cost in Universiti Kebangsaan Malaysia Medical Centre (UKMMC) indicated that the generic drug prescription rate (GDPR) was low (45.2\%) and significant relationship was observed between generic drug prescriptions with age of patients, types of wards and different levels of clinicians' training. It was also stated that the severity of the patient condition, the length of stay, the number of drug prescribed, the type of wards, and the GDPR affect the cost of the drugs [4]. Besides, some clinicians' inability to advocate and educate patients of the same therapeutic properties of generic drugs will lead to escalating cost imposed on government and clients [5-7]. In general, physicians were neutral to slightly supportive on the use of generic drugs. Factors that might influence the physicians view on generic drugs including policy-related issues, patient-related variables, drug characteristics, and physician-related variables. Many physicians still have concerns about the overall quality and reliability of generic drugs as well as generic interchange of certain drug categories [8]. Another study on pharmacists' view indicated that economic, scientific, and policy-related considerations as matters for discussion of generic drugs usage [9].

Malaysia healthcare is public funded where the government is the main provider for the health-care system. National Formulary is the list approved by the government to procure the drugs for hospitals. It is through the selection of drugs to be listed in National Formulary that government could ensure the availability of appropriate drugs to be given to the patients with minimum cost. Drugs listed in UKM Formulary are dispensed to patients with minimum cost while patients are required to pay the full cost to obtain drugs outside the list. The dyslipidemia drugs listed in UKM Formulary for the year of 2012 were lovastatin, ciprofibrate, fenofibrate, and gemfibrozil. The non-formulary dyslipidemia drugs that normally prescribed by medical officers at UKM included rosuvastatin, ezetimibe, simvastatin, and atorvastatin. The 
study of the cost for formulary and non-formulary drugs can be used to compare the effectiveness of budget allocation by the government.

Therefore, this study aims to impute the direct drug cost of patients using dyslipidemia drugs at outpatients' Pharmacy Department of UKMMC. It is through the study of the patients' prescriptions that we will know the factors affecting the drug cost and prescribing pattern for the patients. These could contribute toward short- and long-term strategies to reduce the drug cost expenditure of dyslipidemia drugs in the hospital.

\section{METHODS}

\section{Patients selection}

This study was carried out in UKMMC outpatient Pharmacy Department. The patients were selected from outpatient Pharmacy Information System (PhIS), a database that keeps all the outpatients' prescriptions information in UKMMC. The usage of the database was approved by the Research and Ethics Committee of UKM Medical Centre. The sample size calculation was based on branded drug prescription rate of 55\% and generic prescription rate of $45 \%$ in a study done among cardiovascular patients in UKMMC [4]. A total of 380 patients' prescriptions were calculated to be used in the cost analysis. The ID of the patients who prescribed with at least one dyslipidemia drug from January 1, 2012, to December 31, 2012, was captured from PhIS. A total of 380 patients were randomly selected from the ID using the Fischer random table. The inclusion criteria were patients who prescribed with at least one dyslipidemia drugs in the year 2012 while the exclusion criteria were patients who just discharged from the hospital ward or the patients referred from other hospitals.

\section{Data collection procedures}

Once the ID of the patients was randomly selected, all the details of the prescriptions prescribed from the duration of January 1, 2012December 31, 2012, were traced from the PhIS database. Data collected included ethnicity, age, and gender. The pharmacist-in-charge was interviewed to determine the type of drugs procured and dispensed, the characteristic of the drugs whether the drugs were the originator or generic, formulary or non-formulary.

\section{Analysis of total costs}

The procurement cost for the drugs in Pharmacy Department of UKMMC was captured from PhIS database. Patients' total costs of drugs were calculated by multiplying all drugs dispensed (in the prescriptions received) with the unit cost of each drug. To compare the formulary and non-formulary dyslipidemia drugs cost, the assumption of once daily dosing for non-formulary dyslipidemia drugs was done, and the costs of non-formulary dyslipidemia drugs were calculated by multiplying the assumed quantity of drugs dispensed with the unit cost of each drug. The cost was in Malaysia currency which is Ringgit Malaysia (RM).

\section{Data analysis}

Data extracted from the database were entered into Microsoft Excel for basic data cleaning and analysis before imported into SPSS (Statistical Package for the Social Sciences Version 22) for further data screening and analysis. The normality of the data was tested using KolmogorovSmirnov test. Besides, the skewness and kurtosis values were determined as well. Subsequent tests were chosen according to the normality of the data. Two-tailed test was conducted. The alpha level of significance for all inferential statistics was set at 0.05 unless otherwise specified. For analysis purposes, the following variables were divided into categories. The variables that were divided into different categories included patients age, number of drugs prescribed per patients, number of prescription per patient per year, and GDPR classification.

\section{RESULTS}

\section{Patient demographics}

As shown in Table 1, there were 380 patients accepted for data analysis. There were more male patients as compared to female patients. The number of Malay ethnic was highest, followed by Chinese ethnic, Indian ethnic, and other ethnic. The average age of the patients was 61 .

\section{Prescription characteristics}

As shown in Table 2, the number of prescriptions received by the 380 patients was 829. From the distribution of the number of prescriptions received by each patient, most of the patients received two prescriptions a year followed by one prescription.

The calculated total number of drugs prescribed for 829 prescriptions was 5512. From the amount, 990 were dyslipidemia drugs. For the analysis of formulary and non-formulary dyslipidemia drugs, 748 were formulary dyslipidemia drugs, and 242 were non-formulary drugs. Table 3 show the number of formulary dyslipidemia drugs prescribed. The most number of formulary dyslipidemia drug prescribed was lovastatin $40 \mathrm{mg}$ (46.8\%), followed by lovastatin $20 \mathrm{mg}$ (27.9\%), fenofibrate $160 \mathrm{mg}$ (23.7\%), gemfibrozil $300 \mathrm{mg}(1.1 \%)$, and ciprofibrate $100 \mathrm{mg}(0.5 \%)$ (Table 3$)$.

Table 4 show the number of non-formulary dyslipidemia drugs prescribed. The most number of non-formulary dyslipidemia drugs prescribed was rosuvastatin $10 \mathrm{mg}(22.8 \%)$, followed by atorvastatin $20 \mathrm{mg}(19.4 \%)$ and simvastatin $20 \mathrm{mg}(17.4 \%)$. The least prescribed non-formulary dyslipidemia drugs were simvastatin $10 \mathrm{mg} \mathrm{(2.9 \% )}$ and $40 \mathrm{mg}(2.9 \%)$, followed by atorvastatin $40 \mathrm{mg}(2.5 \%)$ and atorvastatin $80 \mathrm{mg}(0.9 \%)$. Statins group was the most prescribed group in nonformulary dyslipidemia drugs such as rosuvastatin, simvastatin, and atorvastatin which in total contribute $91 \%$ of the total number of nonformulary dyslipidemia drug prescription.

\section{Generic drug prescribing pattern}

For the study of generic drugs prescribed, out of the total number of 5512 drugs prescribed, 3967 (72\%) were generic drugs. The GDPR for each patient was calculated by sum up the total number of generic drug prescribed to each patient and divided by the total drug prescribed for each patient. High GDPR indicates more generic drugs were prescribed to the patient while low GDPR indicate less generic drug was prescribed to the patient. Most of the patients (73.5\%) were having GDPR range from $61 \%$ to $100 \%$. This showed that most of the patients were prescribed with more generic drugs than originator drugs. The range of GDPR versus number of patients was shown in Table 5 .

The GDPR was then categorized into $75 \%$ or less and more than $75 \%$. The number of patients with GDPR of $75 \%$ or less was 197 (52\%) while the number of patients with GDPR of more than $75 \%$ was $183(48 \%)$ (Table 6).

\section{Direct drug cost of patients}

The overall drug cost for all 829 prescriptions was RM240,244.27. The mean cost for each patient was RM637.25 (Range: RM1.20RM27,972.30), and the median cost was RM233.00.

For the study of dyslipidemia drugs, the total formulary dyslipidemia drugs cost prescribed was RM11,002.93. The mean formulary

Table 1: Sociodemographic profile of patients using dyslipidemia drugs in UKMMC

\begin{tabular}{ll}
\hline Characteristics & Frequency n (\%) \\
\hline Gender (n, \%) & \\
Male & $207(54.5)$ \\
Female & $173(45.5)$ \\
Age (mean years and SD) & $61.1 \pm 11.82$ \\
Ethnicity (n, \%) & $175(46.1)$ \\
Malay & $174(45.8)$ \\
Chinese & $26(6.8)$ \\
Indian & $5(1.3)$ \\
Others & \\
\hline
\end{tabular}

UKMMC: Universiti Kebangsaan Malaysia Medical Centre, SD: Standard deviation 
Table 2: Number of prescriptions per patient

\begin{tabular}{lll}
\hline $\begin{array}{l}\text { Number of } \\
\text { prescription }\end{array}$ & $\begin{array}{l}\text { Number of } \\
\text { patients (\%) }\end{array}$ & $\begin{array}{l}\text { Total prescription } \\
\text { (Number of prescription X No. } \\
\text { of patients) (\%) }\end{array}$ \\
\hline 1 & $116(30.5)$ & $116(14.0)$ \\
2 & $164(43.2)$ & $328(39.6)$ \\
3 & $47(12.4)$ & $141(17.0)$ \\
4 & $34(8.9)$ & $136(16.4)$ \\
5 & $9(2.4)$ & $45(5.4)$ \\
6 & $7(1.8)$ & $42(5.1)$ \\
7 & $3(0.8)$ & $21(2.5)$ \\
Total & $380(100.0)$ & $829(100.0)$ \\
\hline
\end{tabular}

Table 3: Number of formulary dyslipidemia drugs prescribed

\begin{tabular}{ll}
\hline Dyslipidemia drugs & Number of drugs prescribed (\%) \\
\hline Lovastatin & \\
$20 \mathrm{mg}$ & $209(27.9)$ \\
$40 \mathrm{mg}$ & $350(46.8)$ \\
Ciprofibrate & \\
$100 \mathrm{mg}$ & $4(0.5)$ \\
Fenofibrate & \\
$160 \mathrm{mg}$ & $177(23.7)$ \\
Gemfibrozil & $8(1.1)$ \\
$300 \mathrm{mg}$ & $748(100.00)$ \\
Total & \\
\hline
\end{tabular}

Table 4: Number of non-formulary dyslipidemia drugs prescribed

\begin{tabular}{ll}
\hline Dyslipidemia drugs & Number of drugs prescribed (\%) \\
\hline Rosuvastatin & \\
$10 \mathrm{mg}$ & $55(22.8)$ \\
$20 \mathrm{mg}$ & $36(14.8)$ \\
Ezetimibe & $13(5.3)$ \\
$10 \mathrm{mg}+$ simvastatin & \\
$20 \mathrm{mg}$ & \\
Ezetimibe $10 \mathrm{mg}$ & $9(3.7)$ \\
Simvastatin & $7(2.9)$ \\
$10 \mathrm{mg}$ & $42(17.4)$ \\
$20 \mathrm{mg}$ & $7(2.9)$ \\
$40 \mathrm{mg}$ & $18(7.4)$ \\
Atorvastatin & $47(19.4)$ \\
$10 \mathrm{mg}$ & $6(2.5)$ \\
$20 \mathrm{mg}$ & $2(0.9)$ \\
$40 \mathrm{mg}$ & $242(100.0)$ \\
$80 \mathrm{mg}$ & \\
Total &
\end{tabular}

dyslipidemia drugs cost for each patient was RM38.07 (Range: RM1.20RM810.00), and the median cost was RM13.50 as shown in Table 7.

As shown in Table 8, the direct drug cost for non-formulary dyslipidemia drugs was estimated to be RM49694.40. Rosuvastatin $10 \mathrm{mg}$ was having the highest non-formulary dyslipidemia drug cost, followed by rosuvastatin $20 \mathrm{mg}$ and atorvastatin $20 \mathrm{mg}$. The direct drug cost for non-formulary dyslipidemia drugs was estimated to be 5 times higher than formulary dyslipidemia drugs.

\section{Comparison of total drug cost with variables}

Mann-Whitney U-test and Kruskal-Wallis Test were conducted to compare the total drug cost with different variables. It was found that there are four variables that significantly incur the higher total cost of drugs in this study. Patients' age group of more than 60 years old $(p<0.05)$, the number of drugs prescribed that are more than 10 drugs $(p<0.05)$, the number of prescription of more than one prescription per patient $(\mathrm{p}<0.05)$, and GDPR of equal or $<75 \%$ incur a higher total drug cost. The results were shown in Table 9.
Table 5: Range of generic drugs prescription rate (GDPR) (\%) versus number of patients

\begin{tabular}{ll}
\hline Range of GDPR (\%) & Number of patients (\%) \\
\hline $0-20$ & $12(3.2)$ \\
$21-40$ & $24(6.3)$ \\
$41-60$ & $65(17.0)$ \\
$61-80$ & $126(33.2)$ \\
$81-100$ & $153(40.3)$ \\
Total & $380(100.0)$ \\
\hline
\end{tabular}

GDPR: Generic drug prescription rate

Table 6: GDPR

\begin{tabular}{ll}
\hline GDPR (\%) & Number of patients (\%) \\
\hline GDPR $\leq 75$ & $197(52)$ \\
GDPR $>75$ & $183(48)$ \\
\hline
\end{tabular}

GDPR: Generic drug prescription rate

Multiple logistic regressions ("Enter" method) were performed to ascertain the effects of patients' age, number of drugs prescribed per patients, number of prescription per patient, and GDPR on the likelihood that patients have a higher cost of more than RM233. It was found that two predictor variables, GDPR $(\leq 75 \%)$ and number of drugs prescribed per patient were statistically significant as shown in Table 10. Number of drugs prescribed for more than 10 had 7 times higher odds to higher total drug cost while GDPR of $75 \%$ or less had 2.18 times higher odds to higher total drug cost.

\section{DISCUSSION}

In this study, $83 \%$ of the male patients were at the 50 years age and above while $84 \%$ of the female patients were at the age of 50 and above. This result was consistent with the National morbidity survey [2], where there is a trend of increase dyslipidemia cases with the increase in age.

From the study of the prescription characteristics, it was found that most of the patients received two prescriptions in a year. The finding of this study proved that there was a significant association between the numbers of prescriptions prescribed with total drug cost. The cost will be higher when the number of prescriptions prescribed increase as more drugs will be prescribed to the patients. However, further study needs to be done to test the level of compliance of the patients given the prescribed drugs to see whether there are any oversupply or non-compliance cases among the patients. The increases in the level of compliance may indirectly help in preventing wastage of the drugs and hence reduce the drug cost.

The previous study in UKMMC showed that the GDPR in inpatient pharmacy of UKMMC was $45.2 \%$ [4]. The low generic drug prescription was caused by very wide range on personal choices. In this study which was done at the same teaching hospital, the GDPR was increased from $45.2 \%$ to $72 \%$. Although the previous study was done in inpatient department and current study was done in the outpatient department, the increase in the generic drug usage can be observed. This may be due to the increased awareness of the generic drug usage especially during drug procurement and drug prescription. In this study, it was found that GDPR was one of the factors that caused the reduction in the total drug cost. Therefore, it is important to promote the use of the generic drug in government facilities as a step to reduce the drug cost from the provider point of view.

Hyperlipidemia, hypertension, and ischemic heart disease were the prevalent disease among the patients with CVD. Most of the time, combinations of drugs were prescribed to the patients for effective therapy [10]. From the study, it was found that there was a wide range of drug cost per patient. This might be caused by the presence of different comorbidities in the patients. All the other comorbidities cases in 
patients with dyslipidemia may contribute to the increase in total drug cost. Further studies can be done to estimate the cost-effectiveness of various drugs given to the patients by monitoring the outcome of the patients through the medical records.

Statins group of dyslipidemia drugs remain as the highest group of drugs being prescribed for outpatients in UKMMC. It was stated that low-density lipoprotein reduction with statins treatment remained the cornerstone of lipid-lowering therapy to reduce the risk ofCVD. To study the pattern of the management of dyslipidemia drug prescribed to the

Table 7: Direct drug cost for formulary dyslipidemia drugs

\begin{tabular}{ll}
\hline Dyslipidemia drugs & Direct drug cost (RM) \\
\hline Lovastatin & \\
$20 \mathrm{mg}$ & 968.08 \\
$40 \mathrm{mg}$ & 2025.75 \\
Ciprofibrate & \\
$100 \mathrm{mg}$ & 801.00 \\
Fenofibrate & \\
$160 \mathrm{mg}$ & 7009.20 \\
Gemfibrozil & 198.90 \\
$300 \mathrm{mg}$ & 11002.93 \\
Total &
\end{tabular}

Table 8: Estimated direct drug cost for non-formulary dyslipidemia drugs

\begin{tabular}{ll}
\hline Dyslipidemia drugs & Direct drug cost (RM) \\
\hline Rosuvastatin & \\
$10 \mathrm{mg}$ & $12,675.30$ \\
$20 \mathrm{mg}$ & 8796.90 \\
Ezetimibe $10 \mathrm{mg}+$ simvastatin & 2392.50 \\
$20 \mathrm{mg}$ (Vytorin) & \\
Ezetimibe $10 \mathrm{mg}$ & 2718.60 \\
Simvastatin & \\
$10 \mathrm{mg}$ & 1449.00 \\
$20 \mathrm{mg}$ & 6948.00 \\
$40 \mathrm{mg}$ & 824.40 \\
Atorvastatin & \\
$10 \mathrm{mg}$ & 2835.00 \\
$20 \mathrm{mg}$ & 8389.80 \\
$40 \mathrm{mg}$ & 1228.50 \\
$80 \mathrm{mg}$ & 1436.40 \\
Total & 49694.40 \\
\hline
\end{tabular}

patients, the patients' lipid profile needed to be studied and recorded to determine the effectiveness of dyslipidemia drugs in controlling patients' dyslipidemia condition.

The most prescribed non-formulary dyslipidemia drug was rosuvastatin $10 \mathrm{mg}$. There was a study suggested that the efficacy of rosuvastatin was more than atorvastatin in lipid-lowering effect and high-density lipoprotein-cholesterol raising effect [11]. However, the condition of the patients and the cost of the drugs still needed to be considered.

The estimated cost for non-formulary dyslipidemia drugs was found to be 5 times higher than the cost of formulary dyslipidemia drugs. The decision on drug listing in formulary found to be a way to reduce direct drug cost. However, there is a need to do studies on drugs for other criteria including safety, effective, and cost-effective before the decision made to list the drugs in the formulary. Multiple criteria decision analysis should be considered for drug listing as well [12].

GDPR and number of drugs prescribed were the variables that were related to the total drug cost. The implementation of generic drug usage policy was found to reduce the cost of the drugs. There was a study done showed that each one percentage point increase in GDPR was associated with a drop of $2.5 \%$ in gross pharmacy expenditure [13]. Besides, the more frequent the outpatients visit the clinic, the more tendency for the medical officers to prescribe more drugs, and hence increase the total drug cost. Therefore, proper planning should be done in deciding the appropriate frequency of visits for outpatients with chronic cases.

There were a few limitations in this study that might be used as a reference in the study the direct drug cost. This study only looked at the providers' direct drug cost but did not take into account the patients' indirect cost. This was due to the reason that most of the dyslipidemia patients were treated with other multiple comorbidity cases and it is inappropriate to calculate the indirect cost to treat dyslipidemia cases alone. To study the trend of the changes in drug cost throughout the years, the prescriptions data should be collected for multiple years. However, due to the limited time and resources, this study only managed to study the drug cost for 1 year. University hospitals are different from Ministry of Health public hospitals in term of management and the process of drug procurement. It is useful to consider obtaining prescriptions data from multiple hospitals to see the trend of procurement in a different hospital. To study the cost-effectiveness of the drugs, it is necessary to refer to patients' medical records to study the suitability of the drugs prescribed and dispensed. Besides, the compliance of the patients

Table 9: Comparison of total drug cost among different variables

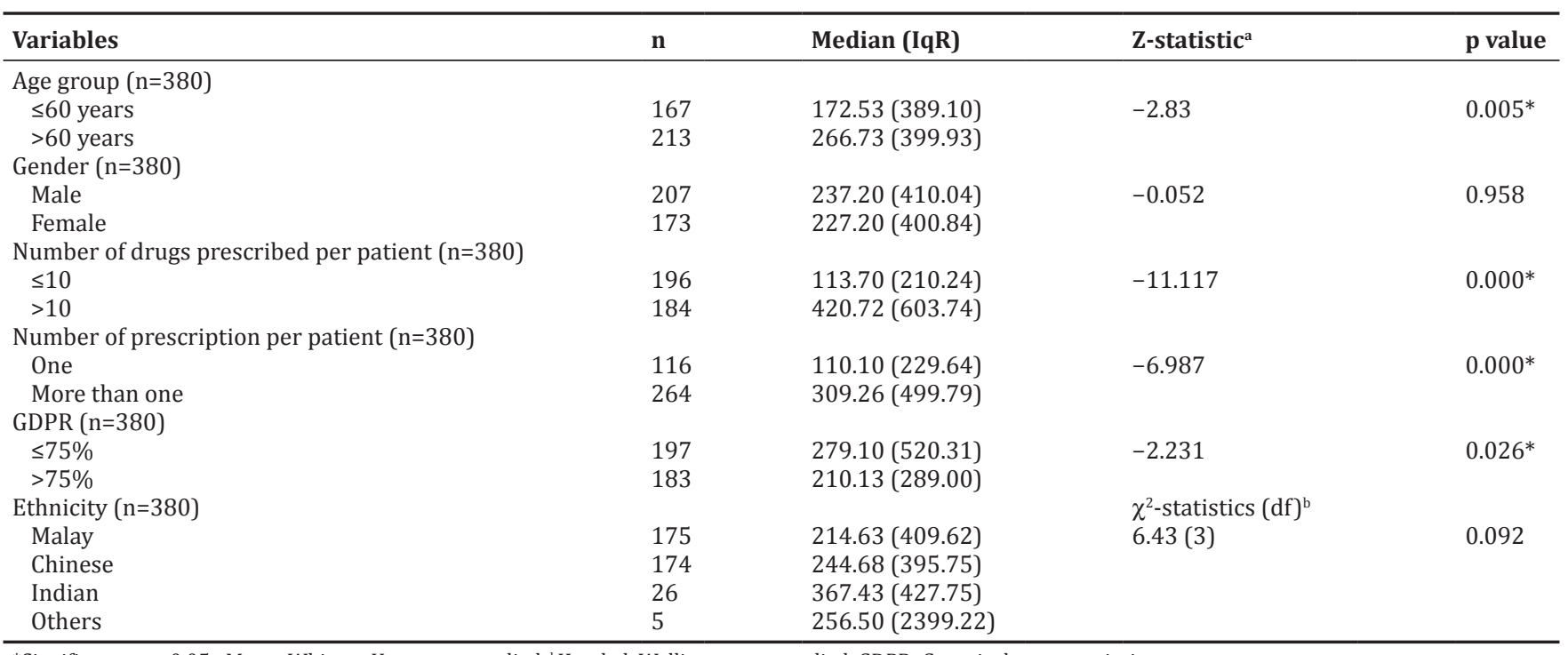

*Significant at $\mathrm{p}<0.05,{ }^{\mathrm{a}} \mathrm{Mann}-$ Whitney U-test was applied, ${ }^{\mathrm{b}}$ Kruskal-Wallis test was applied. GDPR: Generic drug prescription rate 
Table 10: Predictor of total drug cost

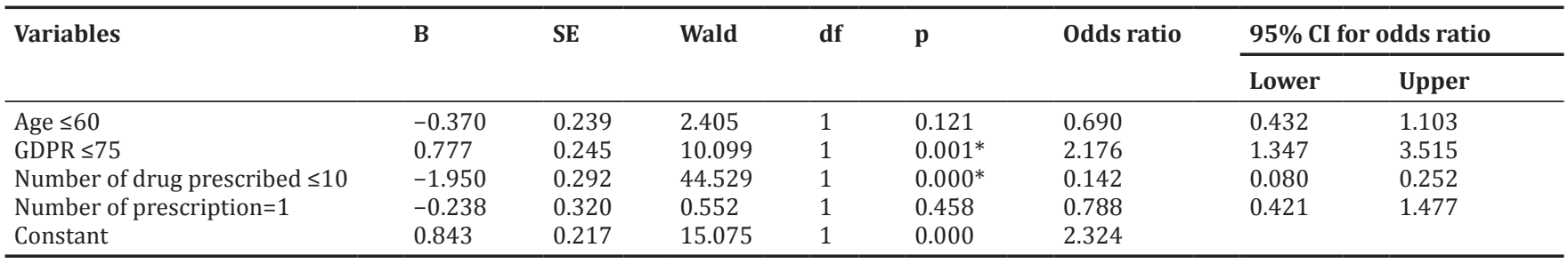

*Significant at $\mathrm{p}<0.05$. SE: Standard error, CI: Confidence intervals

receiving the prescribed drugs should be studied to see its effects on the drug cost.

\section{CONCLUSION}

Although there is much debate on the usage of generic drugs in reducing the cost of the drugs, it is generally accepted that generic drugs substitution helps in reducing overall drug cost. It was a challenge to maintain the bioequivalence of generic drugs with the originator's drug to provide the safety, effective, and cost-effective drugs for the patients. Besides, training and encouragement should be given to the prescribers to prescribe the generic drug. The big difference of the estimated expenditure of non-formulary drugs as compared to the formulary drugs indicates that the proper workflow and criteria should be set up to decide the listing of the drugs in the National Formulary.

\section{ACKNOWLEDGMENT}

Our deep appreciation and gratitude for UKM Medical Centre, especially staffs in outpatients pharmacy department for giving valuable support and cooperation to facilitate this study and provided the necessary information for this study. We would also like to thank Research and Ethics Committee of UKM Medical Centre for the approval to conduct this study.

\section{REFERENCES}

1. Ministry of Health Malaysia. Clinical Practice Guidelines Management of Dyslipidemia. $4^{\text {th }}$ ed. Putrajaya: Ministry of Health Malaysia; 2011.
2. Ministry of Health Malaysia. National Health and Morbidity Survey 2015. Putrajaya: Institute for Public Health, Ministry of Health; 2015.

3. Ministry of Health Malaysia. Malaysian National Medicines Policy. $2^{\text {nd }}$ ed. Putrajaya: Ministry of Health Malaysia; 2012.

4. Al-Junid SM, Ezat WP, Surianti S. Prescribing patterns and drug cost among cardiovascular patients in hospital Universiti Kebangsaan Malaysia. Med J Malaysia 2007;62:59-65.

5. Copeland C. Prescription drugs: Issues of cost, coverage, and quality. EBRI Issue Brief 1999;208:1-21.

6. Lucena González MI, Ruiz Ruiz J, Andrade Bellido RJ, Hidalgo Sánchez R, González-Correa JA, García Ruiz A, et al. The impact of hospitalization on drug prescription. Med Clin (Barc) 1995;104:211-5.

7. Litton LM, Sisk FA, Akins ME. Managing drug costs: The perception of managed care pharmacy directors. Am J Manag Care 2000;6:805-14.

8. Azmi MH, Akmal SA, Ahmed A, Izham IM, Chong, CP. Physicians' views on generic medicines: A narrative review. J Generic Med 2010;7:30-9.

9. Nabil A, Azmi, MH. Pharmacits' view on generic medicines: A review of the literature. J Generic Med 2008;5:209-18.

10. Mukesh K, Vicky D, Shruti M, Dinesh S, Neha M, Mangla L. Cardiovascular disease prevalence and drug utilization patterns at a tertiary care hospital in Northeastern India. Int J Pharm Pharm Sci 2016;8:116-9.

11. Mohammad AK, Krishna M, Vaibhav G, Kanhaya L, Dharmendra S, Pradeep D, et al. Atorvastatin VS rosuvastatin; fenofibrate as an add on: An exploratory study. Int J Pharm Pharm Sci 2014;6:493-8.

12. Thokala P, Duenasm A. Multiple criteria decision analysis for health technology assessment. Value Health 2012;15:1172-81.

13. Liberman JN, Roebuck MC. Prescription drug costs and the generic dispensing ratio. J Manag Care Pharm 2010;16:502-6. 